\section{Overview of the 2013 ACC/AHA Guideline on the Treatment of Blood Cholesterol to Reduce Atherosclerotic Cardiovascular Risk in Adults}

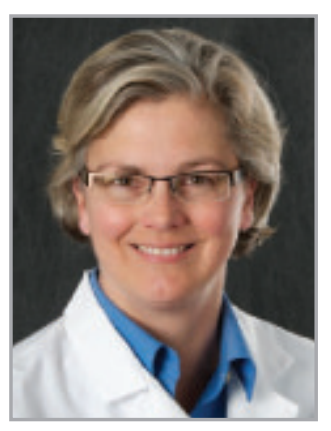

“The 2013 ACC/AHA cholesterol guideline provides

a comprehensive, evidence-based approach to

reducing atherosclerotic cardiovascular

disease risk.”

Jennifer GRobinson*

The recently published 2013 ACC/AHA Guideline on the Treatment of Blood Cholesterol to Reduce Atherosclerotic Cardiovascular Risk in Adults published by the American Heart Association (AHA) and American College of Cardiology (ACC) changes the treatment paradigm for cholesterol treatment [1]. Based on a rigorous, systematic review of randomized controlled clinical trials (RCTs), the new guideline no longer targets LDL cholesterol (LDL-C) levels. The new recommendations now focus on treating cholesterol to reduce atherosclerotic cardiovascular disease (ASCVD) risk. The 2013 guideline approach is consistent with the 2011 report of the Institute of Medicine (IOM) on trustworthy guidelines [2]. Only data from RCTs of cholesterol-lowering drug therapies with cardiovascular outcomes, or meta-analyses of these RCTs, were considered. RCTs are the most objective assessment of cardiovascular event reduction benefits and adverse effects that can be used to guide clinical practice. The cholesterol guideline effort was supported by the lifestyle and risk assessment working groups, which undertook a similarly rigorous process to developing their evidence-based recommendations [3,4].

\section{Recommendations}

Because the focus is on the evidencebased treatment of cholesterol to reduce ASCVD risk in adults, the recommendations emphasize statin therapy. A very large body of evidence from multiple populations is available for statins, with less evidence available for nonstatin drug therapy for ASCVD risk reduction.

- A healthy lifestyle is the foundation for ASCVD risk reduction

The RCTs were performed in the setting of healthy lifestyle advice. Long-term adherence to healthy lifestyle habits and control of other ASCVD risk factors is an essential component of ASCVD risk-reduction efforts. The cholesterol panel endorsed the recommendations of the 2013 AHA/ACC Guideline on Lifestyle Management to Reduce Cardiovascular Risk [3].

*Department of Epidemiology, 145 N Riverside Dr S455 CPBH, University of lowa, lowa City, IA 52242, USA;

\section{KEYWORDS}

- cholesterol • evidence-based

- guideline $\bullet$ prevention $\bullet$ systematic review

\section{"Based on a rigorous, systematic review of randomized controlled clinical trials, the new guideline no longer targets LDL cholesterol levels."}


"Strong randomized controlled clinical trial evidence supports a net benefit from statin therapy in individuals with clinical atherosclerotic cardiovascular disease (secondary prevention)...”
- Use statin therapy to reduce ASCVD risk in those most likely to benefit

Strong RCT evidence supports a net benefit from statin therapy in individuals with clinical ASCVD (secondary prevention), and in three groups of individuals without clinical ASCVD (primary prevention), age $\geq 21$ years with LDL-C $\geq 190 \mathrm{mg} / \mathrm{dl}$, diabetes aged $40-75$ years or $\geq 7.5 \%$ 10-year ASCVD risk aged 40-75 years with LDL-C 70-189 mg/dl. Moderate evidence supports the use of a statin for primary prevention in those with 5-<7.5\% 10-year ASCVD risk aged 40-75 years with LDL-C 70-189 mg/dl. Statin therapy may also be considered in those who do not meet these criteria if other indications of increased ASCVD risk are present. For those in whom a risk-based decision is unclear, an LDL-C $\geq 160 \mathrm{mg} / \mathrm{dl}$, family history of premature ASCVD (first-degree male relatives before age 55 years or female relatives before age 65 years), high lifetime ASCVD risk, elevated coronary artery calcium, high-sensitivity CRP $\geq 2 \mathrm{mg} / \mathrm{l}$ or an ankle-brachial index $<0.9$ may indicate increased ASCVD risk. Statins should not be routinely initiated in individuals with New York Heart Association (NYHA) heart failure class II-IV, or in those receiving maintenance hemodialysis due to a lack of an ASCVD risk-reduction benefit.

\section{- Statins are safe in properly selected} individuals when appropriately monitored Statins have an excellent record of safety in RCTs, and there is no longer any reason to monitor hepatic transaminases or creatine kinase unless clinically indicated due to symptoms. In general, the RCTs enrolled individuals who were without serious cormorbid conditions. Trial participants were regularly monitored for safety as well as efficacy (see more on monitoring in section 'Do not titrate cholesterol-lowering drug therapy to LDL-C or non-HDL-C goals'). Statin intensity (see section 'Choose the appropriate intensity of statin therapy to reduce ASCVD risk') should be adjusted in individuals with an increased risk of statin adverse effects (age $>75$ years, a history of statin intolerance, or other characteristics or drug therapy that may influence statin safety).

Muscle symptoms are common, but not necessarily related to statin use. The relationship of muscle or other symptoms to statin therapy should be established by discontinuing the statin, then rechallenging with the original or lower dose of the same or a different statin. Statin-related severe myopathy (with creatine kinase elevations), rhabdomyolysis and hemorrhagic stroke are rare.

Statins modestly increase the risk of diabetes, but the ASCVD risk-reduction benefit exceeds the risk of diabetes in all but the lowestrisk individuals. Screening for diabetes should occur according to current diabetes guidelines. Healthy lifestyle habits should be encouraged in all patients.

\section{- A clinician-patient discussion prior to statin initiation is important, especially in lower-risk primary prevention}

Estimation of 10-year ASCVD risk is the first step in evaluating the potential for an ASCVD risk-reduction benefit from statin therapy used for primary prevention (2013 Prevention Guidelines Tools CV Risk Calculator using the Pooled Cohort Equations can be found at [5-7]). The Pooled Cohort Equations should be used to estimate risk in white and African-American women and men who are not receiving statin therapy and have an LDL-C $<190 \mathrm{mg} / \mathrm{dl}$. In those with diabetes, 10-year ASCVD risk can now be estimated using the new equations, and may inform the choice of statin intensity. Do not estimate 10 -year risk in those with clinical ASCVD or with LDL-C $\geq 190 \mathrm{mg} / \mathrm{dl}$ because all such adults $\geq 21$ years of age should receive statin therapy.

Once 10-year ASCVD risk has been estimated, the clinician and patient should then engage in a discussion of the potential for an ASCVD riskreduction benefit based on estimated 10-year ASCVD risk and other factors noted in the section 'Use statin therapy to reduce ASCVD risk in those most likely to benefit', adverse effects, drug-drug interactions and patient preferences when deciding whether to initiate statin therapy. The discussion also provides the opportunity to address healthy lifestyle habits and control of other risk factors. After age 75 years, there is less evidence for primary prevention and decisions should be individualized in this age group.

\section{- Choose the appropriate intensity of statin therapy to reduce ASCVD risk}

High-intensity statins lower LDL-C by $\geq 50 \%$ (atorvastatin $40-80$ or rosuvastatin $20-40 \mathrm{mg}$ ). Moderate-intensity statins lower LDL-C by $30-<50 \%$ (atorvastatin 10-20, fluvastatin 80, lovastatin 40, pitvastatin 2-4, pravastatin 40, rosuvastatin $5-10$ or simvastatin $20-40 \mathrm{mg}$ ). 
Atorvastatin 80 , rosuvastatin 20 , atorvastatin 10 , lovastatin 40 , pravastatin 40 and simvastatin 20-40 mg were evaluated in the RCTs with an ASCVD event-reduction benefit. In one trial, atorvastatin $80 \mathrm{mg}$ was downtitrated to $40 \mathrm{mg}$ if symptoms occurred. In secondary prevention, strong evidence supports the use of high-intensity statin therapy for individuals with clinical ASCVD who are $\leq 75$ years of age, unless there are conditions influencing safety. Strong evidence also supports the use of moderate-intensity statin therapy in individuals with clinical ASCVD aged $>75$ years, or if safety concerns are present for high-intensity statin therapy.

In primary prevention, strong evidence supports the use of moderate-intensity statins in individuals with diabetes aged 40-75 years and in those with $\geq 7.5 \% 10$-year ASCVD risk. Moderate evidence supports the use of high-intensity statins only in those with $\geq 7.5 \%$ 10 -year ASCVD risk (with or without diabetes) and moderate intensity statins in those with 5 to $<7.5 \%$ 10-year ASCVD risk and LDL-C 70-189 mg/dl. High-intensity statins should be used in those with LDL-C $\geq 190 \mathrm{mg} / \mathrm{dl}$; addition of nonstatin therapy can be considered when LDL-C levels remain unacceptably elevated (see section 'Regularly monitor adherence \& safety, including LDL-C measurement').

\section{- Do not titrate cholesterol-lowering drug therapy to LDL-C or non-HDL-C goals}

Because the RCTs to date have used fixed doses of statin and/or nonstatin drugs, there is insufficient evidence to recommend titration to specific LDL-C or non-HDL-C goals. Use of such goals has the potential to result in inappropriate therapy. For example, a patient with clinical ASCVD may have an LDL-C $<100 \mathrm{mg} / \mathrm{dl}$ on pravastin $10 \mathrm{mg}$ daily, yet this intensity of statin therapy provides less than optimal therapy for ASCVD prevention. Or, a patient may have an LDL-C of $105 \mathrm{mg} / \mathrm{dl}$ on a high-intensity statin. There is no evidence that addition of a nonstatin to high (or even moderate)intensity statin therapy will further reduce ASCVD risk, nor is there evidence that such an approach has an acceptable margin of safety.

\section{- Regularly monitor adherence \& safety, including LDL-C measurement}

All RCTs regularly evaluated drug adherence and safety. A follow-up visit should be performed within 4-12 weeks of statin initiation or changes in therapy, and should include a fasting lipid panel and assessment of adherence and safety. Percentage reduction in LDL-C consistent with statin intensity may provide a guide to the anticipated response to therapy: approximately $\geq 50 \%$ for a high-intensity statin, or approximately 30-50\% for a moderate-intensity statin. When baseline LDL-C is unknown, it is noted that an LDL-C $<100 \mathrm{mg} / \mathrm{dl}$ was achieved by most participants receiving a high-intensity statin. Percentage reduction or LDL-C level should never be used as performance measures.

If a less-than-anticipated response occurs, improved adherence to lifestyle and drug therapy should be encouraged, and/or statin therapy can be increased. Rule out secondary causes of hypercholesterolemia if indicated (most common causes are weight gain or obesity, high saturated or trans-fat intake, hypothyroidism, biliary obstruction or pregnancy).

In those receiving maximally tolerated statin therapy but still having an unacceptable response to therapy, addition of a nonstatin therapy can be considered in high-risk individuals if the ASCVD risk-reduction benefits are considered to outweigh the increased risk of adverse effects. High-risk individuals include those with clinical ASCVD, LDL-C $\geq 190 \mathrm{mg} / \mathrm{dl}$ or diabetes aged 40-75 years and LDL-C 70-189 mg/dl.

Once a stable lifestyle and drug therapy regimen has been established, follow-up should occur at 3-12-month intervals.

\section{Summary}

The 2013 ACC/AHA cholesterol guideline provides a comprehensive, evidence-based approach to reducing ASCVD risk. As with all guidelines, these recommendations require consideration of the potential net benefit for each patient, based on their unique characteristics and preferences.

Financial \& competing interests disclosure JG Robinson was the Vice Chair for the 2013 ACC/AHA cholesterol guideline. In the past year, the author has received research grants from Amarin, Amgen, AstraZeneca, Daiichi Sankyo, Esperion, Genentech/Hoffmann-La Roche, GlaxoSmithKline, Merck, Regeneron/Sanofi andZinfandel/ Takeda, and has served as consultant for Amgen, Hoffmann-La Roche, Pfizer and Sanofi. The author has no other relevant affiliations or financial involvement with any organization or entity with a financial interest in or financial conflict with the subject matter or materials discussed in the manuscript apart from those disclosed.

No writing assistance was utilized in the production of this manuscript.
"In those receiving maximally tolerated statin therapy but still having an unacceptable response to therapy, addition of a nonstatin therapy can be considered in high-risk individuals..." 


\section{References}

1 Stone N, Robinson J, Lichtenstein A et al. 2013 ACC/AHA Guideline on the Treatment of Blood Cholesterol to Reduce

Atherosclerotic Cardiovascular Risk in Adults: a Report of the American College of Cardiology/American Heart Association Task Force on Practice Guidelines. Circulation doi:10.1161/01.cir.0000437738.63853.7a (2013) (Epub ahead of print).

2 Committee on Standards for Developing Trustworthy Clinical Practice Guidelines. Clinical Practice Guidelines We Can Trust. Institute of Medicine, DC, USA (2011)
3 Eckel RH, Jakicic JM, Ard JD et al. 2013 AHA/ACC Guideline on Lifestyle Management to Reduce Cardiovascular Risk: a Report of the American College of Cardiology/American Heart Association Task Force on Practice Guidelines. Circulation doi:10.1161/01.cir.0000437740.48606.d1 (2013) (Epub ahead of print).

4 Goff DC, Lloyd-Jones DM, Bennett G et al. 2013 ACC/AHA Guideline on the Assessment of Cardiovascular Risk: a Report of the American College of Cardiology/ American Heart Association Task Force on Practice Guidelines. Circulation doi:10.1161/01.cir.0000437741.48606.98 (2013) (Epub ahead of print).

5 American Heart Association. 2013 Prevention Guidelines Tools. CV Risk Calculator (iTunes version).

https://itunes.apple.com/us/app/ascvd-riskestimator/id808875968?mt=8\&ign$\mathrm{mpt}=\mathrm{uo} \% 3 \mathrm{D} 2$

6 ASCVD Risk Estimator (Android version). https://play.google.com/store/apps/ details?id $=$ org. acc. cvrisk $\& h l=$ en

7 ASCVD Risk Estimator* (desktop version). http://tools.cardiosource.org/ASCVD-RiskEstimator 